medication refill $(n=15)$ with faxed $(n=14)$ or mailed $(n=13)$ prescriptions. Fourteen clinics had staff redeployed to assist the COVID-19 response; 14 clinics reported a reduction in total number of full-time equivalent (FTE) clinical nurses from 74.4 to 45.6 FTE collectively and three clinics reported reduction in FTE clinical doctors, from 20.1 to 17.1 FTE collectively.

Conclusion Australian public sexual health clinics rapidly pivoted service delivery to reduce the risk of COVID-19 transmission in their clinical settings, managed staffing reductions and delays in molecular testing, released staff to support the COVID-19 response, and maintained a focus on urgent and symptomatic STI presentations and those at higher risk of HIV/STI acquisition.

\section{P272 AGE PATTERNS OF ORAL AND ANAL SEXUAL PRACTICES AMONG HETEROSEXUAL MALES AND FEMALES: A CROSS-SECTIONAL SURVEY IN MELBOURNE, AUSTRALIA}

1,2 $\mathrm{T}_{\text {Phillips }}{ }^{*},{ }^{1,2} \mathrm{H}$ Constantinou, ${ }^{1,2} \mathrm{C}$ Fairley, ${ }^{1,2} \mathrm{C}$ Bradshaw, ${ }^{2} \mathrm{~K}$ Maddaford, ${ }^{1,2} \mathrm{M}$ Chen, ${ }^{3} \mathrm{~J}$ Hocking, ${ }^{1,2,3} \mathrm{E}$ Chow. ${ }^{1}$ Monash University, Clayton, Australia; ${ }^{2}$ Melbourne Sexual Health Centre, Carlton, Australia; ${ }^{3}$ The University of Melbourne, Parkville, Australia

10.1136/sextrans-2021-sti.343

Background Oral and anal sex practices among heterosexuals are not well-studied. We aimed to explore these sexual practices among heterosexuals attending a sexual health clinic.

Methods This cross-sectional survey was conducted at Melbourne Sexual Health Centre between March-April 2019. This survey asked questions on oral sex (fellatio or cunnilingus), anal sex and rimming in the previous 3 months among heterosexuals. Age was categorized by: $18-24 ; 25-34$; and $\geq 35$ years.

Results There were 709 participants (333 males; 376 females) who were eligible and completed the survey, with a median age of 26 (IQR: 23-31) and 35\% $(\mathrm{n}=250)$ born in Australia. Most participants had had vaginal sex $(n=677 ; 95.5 \%)$, with a median of 2 (IQR: 1-3) vaginal sex partners, and half did not use a condom $(\mathrm{n}=358 ; 50.1 \%) .148$ (20.8\%) participants had had anal sex, with a median of 1 (IQR: 1-1) anal sex partner, with $63.5 \%(n=94)$ not using a condom. There were no significant differences in vaginal/anal sex partner number or condom use by gender. Being $\geq 35$ years was associated with higher mean partner number for anal sex (ptrend $=0.021$ ) and being 18-24 years with higher mean partner number for vaginal sex (ptrend $=0.027)$. Most participants $(n=637$, $89.8 \%$ ) had received oral sex; this proportion did not differ by age group or gender. Females $(n=351,93.4 \%)$ were more likely to perform oral sex than males $(n=275 ; 82.6 \%$ males) $(\mathrm{p}<.001)$. Females were more likely to have received rimming $(26.6 \%$ females vs $12.6 \%$ males; $\mathrm{p}<.001)$ and males were more likely to have performed rimming $(25.5 \%$ males vs $9.3 \%$ females; $\mathrm{p}<.001)$. Performing but not receiving rimming increased with age (ptrend=0.011).

Conclusion Rimming and anal sex are practiced by one fifth or more of heterosexuals. Younger heterosexuals had higher numbers of vaginal sex partners, while older heterosexuals had higher numbers of anal sex partners and were more likely to perform rimming.

\section{P273 SOCIAL NETWORK DISTRIBUTION OF SYPHILIS SELF- TESTING AMONG MSMS IN CHINA: STUDY PROTOCOL FOR A CLUSTER RANDOMIZED CONTROL TRIAL}

${ }^{1,2,3}$ Y Wang* ${ }^{*}{ }^{4} \mathrm{~W}$ Zhang, ${ }^{2} \mathrm{D}$ Bao, ${ }^{5,6} \mathrm{~J}$ Ong, ${ }^{4,6,7} \mathrm{~J}$ Tucker, ${ }^{8} \mathrm{R}$ Ye, ${ }^{1,2,3} \mathrm{H}$ Zheng, ${ }^{1,2,3} \mathrm{~B}$ Yang,

${ }^{1,2,3} \mathrm{C}$ Wang. ${ }^{1}$ Dermatology Hospital of Southern Medical University, Guangzhou, China; ${ }^{2}$ Southern Medical University Institute for Global Health and Sexually Transmitted Diseases, Guangzhou, China; ${ }^{3}$ Guangdong Provincial Center for Skin Disease and STI Control, Guangzhou, China; ${ }^{4}$ University of North Carolina at Chapel Hill, Project-China, Guangzhou, China; ${ }^{5}$ Faculty of Infectious and Tropical Diseases, London School of Hygiene and Tropical Medicine, London, UK; ${ }^{6}$ Central Clinical School, Monash University, Victoria, Australia: ${ }^{7}$ Institute for Global Health and Infectious Diseases, School of Medicine, University of North Carolina at Chapel Hill, Chapel Hill, USA; ${ }^{8}$ Department of Biostatistics, Southern Medical University, Guangzhou, China

10.1136/sextrans-2021-sti.344

Background Syphilis is a common sexually transmitted infection (STI) among men who have sex with men (MSM). Increasing syphilis testing is important to syphilis control. However, in low- and middle-income countries like China, syphilis testing rates remain low among MSM. We describe a randomized controlled trial protocol to examine the effectiveness of social network distribution approaches of syphilis selftesting among MSM in China.

Methods We will recruit index and alter MSM. Indexes will be eligible if they: are born biologically male; aged 18 years or above; ever had sex with another man; are willing to distribute syphilis testing packages or referral links to their alters; and willing to provide personal contact information for future follow-up. Three hundred MSM will be recruited and randomly assigned in a 1:1:1 ratio into three arms: standard of care (control arm); standard syphilis self-testing (SST) delivery arm; and referral link SST delivery arm. Indexes will distribute SST packages or referral links to encourage alters to receive syphilis testing. All indexes will complete a baseline survey and a 3-month follow-up survey. Syphilis self-test results will be determined by photo verification via a digital platform. The primary outcome is the mean number of alters who returned verified syphilis testing results per index in each arm.

Discussion The trial findings will provide practical implications in strengthening syphilis self-testing distribution and increasing syphilis testing uptake among MSM in China. This study also empowers MSM community in expanding syphilis testing by using their own social network.

\section{P274 LESSONS LEARNED FROM AN EDUCATIONAL INTERVENTION TO IMPROVE HIV TESTING BY GPS IN AMSTERDAM, THE NETHERLANDS}

${ }^{1} \mathrm{~S}$ Bogers*, ${ }^{1,2} \mathrm{M}$ Schim van der Loeff, ${ }^{3} \mathrm{~N}$ van Dijk, ${ }^{2} \mathrm{M}$ Groot Bruinderink, ${ }^{1,4} \mathrm{G}$ de Bree, 1,4,5P Reiss, 'S Geerlings, 3,6J van Bergen. 'Department of Internal Medicine, Division of Infectious Diseases, Amsterdam University Medical Centers, location Academic Medical Center, Amsterdam, The Netherlands; ${ }^{2}$ Department of Infectious Diseases, Public Health Service of Amsterdam, Amsterdam, The Netherlands; ${ }^{3}$ Department of General Practice, Amsterdam University Medical Centers, location Academic Medical Center, Amsterdam, The Netherlands; ${ }^{4}$ Amsterdam Institute for Global Health and Development, University of Amsterdam, Amsterdam, The Netherlands; ${ }^{5}$ HIV Monitoring Foundation, Amsterdam, The Netherlands; ${ }^{6}$ STI AIDS Netherlands, Amsterdam, The Netherlands

10.1136/sextrans-2021-sti.345

Background In the Netherlands, general practitioners (GPs) diagnose $79 \%$ of STIs and $36 \%$ of HIV infections, but 